\title{
LIMITS ON THE PRODUCTION OF NEUTRAL PENETRATING STATES IN A BEAM DUMP
}

\author{
J. LoSECCO and L. SULAK \\ University of Michigan, Ann Arbor, MI 48109, USA
}

R. GALIK, J. HORSTKOTTE, J. KNAUER and H.H. WILLIAMS

University of Pennsylvania, Philadelphia, PA 19174, USA

and

\author{
A. SOUKAS, P. WANDERER and W. WENG \\ Brookhaven National Laboratory, Upton L.I., NY 11973, USA
}

Received 17 November 1980

\begin{abstract}
We present limits on the production of neutral penetrating states produced in $28 \mathrm{GeV}$ proton nucleus collisions. We obtain limits for light, heavy and unstable neutral states. For light stable states our limit $\sigma\left[\sigma \mathrm{p}<2.26 \times 10^{-69} \mathrm{~cm}^{4} / \mathrm{nucleon}^{2}\right.$ is more than a factor of 5.5 better than previous limits. Time of flight techniques are used to study heavy states. We have poor sensitivity to short-lived states.
\end{abstract}

There is still a great deal of interest in looking for reasonably light, semistrongly interacting particles e.g. axions [1]. Such particles play a fundamental role in some gauge theories [2]. Neutral heavy particles are also of interest.

We report here on results derived from a beam dump experiment [3] carried out at the Brookhaven AGS. In the experiment a large, 30 ton, liquid scintillator calorimeter [4] was used to observe the particles emerging in the forward direction from $4.9 \times 10^{18}$ $28 \mathrm{GeV}$ protons incident on a beam dump. The dump consisted of a copper block followed immediately by a $30 \mathrm{~m}$ steel muon shield. The dump was located $105 \mathrm{~m}$ from the detector. Fiducial cuts limited the effective detector size to a meter off the beam axis in horizontal and vertical direction $\left(4 \mathrm{~m}^{2}\right)$.

Two other groups [5] ran concurrently with us and have reported results.

The data was analyzed looking for a neutrino signal and any other beam associated signal. Neutrino type events were classified as charged current candidates if they had an energetic penetrating track, a muon signature. All other events above $E_{\text {visible }}>160 \mathrm{MeV}$, were
Table 1

Summary of event statistics

\begin{tabular}{|c|c|c|}
\hline & Bare target & Dump \\
\hline protons & $1.97 \times 10^{17}$ & $4.87 \times 10^{18}$ \\
\hline charged current & 115 & 90 \\
\hline $\begin{array}{l}\text { neutral current } \\
\text { neutral current }\end{array}$ & 24 & 14 \\
\hline charged current & $0.21 \pm 0.05$ & $0.16 \pm 0.04$ \\
\hline total events & 139 & 104 \\
\hline
\end{tabular}

classified as neutral current candidates. Background was found to be negligible.

In addition to the dump exposure, a sample of neutrino events from a $15 \mathrm{~cm}$ thick bare target exposed to $2 \times 10^{17}$ protons was studied. The results of both runs are shown in table 1 .

Heavy particles. The experiment employs a time of flight technique over a distance of $105 \mathrm{~m}$. The time is calibrated both with an independent, bare target, source of ncutrinos and with the neutrinos produced 
from the prompt decay of conventional particles in the dump. Neutrinos arrive at the detector in a "bunch" with fwhm of $30 \mathrm{~ns}$. Stable heavy neutral leptons would produce late events because their travel time to the detector would be slower. They would also probably change the apparent ratio of neutral-current to charged-current events.

The timing distribution from the bare target run indicates only one out-of-time candidate. For the dump run three neutral-current events lie outside of the peak. Two of these are greater than 120 ns late and one is greater than $70 \mathrm{~ns}$. All have energies below $300 \mathrm{MeV}$ and are consistent with beam associated neutrons.

These events were used to predict a subtraction of one event from the peak and are ignored in the subsequent analysis.

With this noted exception we see no evidence for particle interactions later than $40 \mathrm{~ns}$ of the central neutrino induced peak. This gives an upper limit on the time-of-flight for such particles.

$\Delta t / t=-\Delta \beta / \beta, \quad \beta \equiv v / c>0.89$.

This can be converted to a limit on the production of neutral leptons in $28 \mathrm{GeV} / c$ hadronic interactions with

$E_{\text {lepton }} / M_{\text {lepton }}<2.2$.

Since no leptons below the bound (2) are observed but 104 neutrino interactions are observed we find a limit on the coupling, $\sigma_{\mathrm{p}} \sigma_{\mathrm{I}}$ production times scattering cross section for slow heavy leptons

$\sigma_{\mathrm{p}} \sigma_{\mathrm{I}} /\left(\sigma_{\mathrm{p}} \sigma_{\mathrm{I}}\right) \nu_{\text {prompt }}<2.2 \times 10^{-2} \quad(90 \%$ c.l.).

The same detector observed 139 neutrino interactions from an exposure of $2 \times 10^{17}$ protons on a bare target followed by a $60 \mathrm{~m}$ decay space. The limit relative to this standard source is:

$\sigma_{p} \sigma_{I} /\left(\sigma_{p} \sigma_{I}\right)_{\nu}<6.7 \times 10^{-4}$.

Interpretation of eqs. (3) and (4) depends on a model of lepton production and the geometric detector acceptance.

In addition to looking for indications of late arriving particles one can look for correlations of energy with time-of-arrival.

No correlation of energy with time is observed for the beam dump induced events.
Light neutral particles. To get a limit on the production of light neutral particles we consider the number of neutral-current induced interactions. The presence of neutral penetrating particles would produce an enhancement of apparent neutral-current interactions. We will normalize our neutral-current rate to the observed charged-current rate. From the $24 \mathrm{NC}$ interactions in the bare target run and the $115 \mathrm{CC}$ and $90 \mathrm{CC}$ events in the bare target and dump runs, respectively, we would expect $18.8 \pm 4.7 \mathrm{NC}$ events in the dump. We observe $14 \mathrm{NC}$ events in the dump run so the excess is:

excess $\mathrm{NC}=-4.8 \pm 6.0$.

The error quoted is statistical. Systematic errors such as flux measurement or detection efficiency cancel from the prediction. Assuming the error above is gaussian we can get a $90 \%$ c.l. upper limit on the presence of extra neutral interactions ( $1.28 \sigma$ for a one tailed test).

$N_{\text {excess }}<2.9$ events $90 \%$ c.l.

The sensitivity of the experiment is determined by the detector mass ( 11 tons fiducial); the beam exposure $4.8 \times 10^{18}$ protons and the geometric acceptance of the detector, $4 \mathrm{~m}^{2}$ at a distance of $105 \mathrm{~m}$ in front of the dump. As argued in our earlier paper [3] the effective absorption length of the dump is taken as 29 $\mathrm{cm}$ because of the effect of hadron cascades and secondary interactions. The primary absorption length in copper is $15 \mathrm{~cm}$. We will also assume that for a weakly interacting particle $o(A)=A \sigma(A=1)$; there is no nuclear shadowing. As we shall see this assumption is confirmed by the low limit obtained for $\sigma$.

Then the following limit is obtained:

$\eta \sigma_{\mathrm{p}} \sigma_{\mathrm{I}}<2.26 \times 10^{-71} \mathrm{~cm}^{4} /$ nucleon $^{2}$,

$\sigma_{p} o_{I}$ are the interaction and production cross section. $\eta$ is the geometrical acceptance [6] of the detector which depends on the production mechanism.

We have considered a number of production mechanisms. The Bourquin-Gaillard model [7] has been used to study the production of $\pi_{0}$ 's at this energy. If the light neutral is produced like $\pi_{0}$ 's and does not decay, then:

$\eta_{\mathrm{BG}}=0.10$,

$\sigma_{\mathrm{I}} \sigma_{\mathrm{p}}<2.26 \times 10^{-70} \mathrm{~cm}^{4} /$ nucleon $^{2} \quad(90 \%)$. 
If the state is produced isotropically in the center-ofmass:

$\eta_{\mathrm{CM}}=4.2 \times 10^{-4}$,

$\sigma_{\mathrm{I}} \sigma_{\mathrm{p}}<5.38 \times 10^{-68} \mathrm{~cm}^{4} /$ nucleon $^{2} \quad(90 \%)$.

If the state is diffractively produced with a limiting transverse momentum of $P_{\perp}<300 \mathrm{MeV} / c$ and longitudinal momentum of $3 \mathrm{GeV} / c$

$\eta_{\mathrm{D}} \approx 0.05$,

$\sigma_{1} \sigma_{p}<4.52 \times 10^{-70} \mathrm{~cm}^{4} /$ mucleon $^{2} \quad(90 \%)$.

These limits assume that the state will penetrate the $30 \mathrm{~m}$ stecl shield without attenuation and will not decay in flight to the detector.

The time of flight for relativistic particles to the detector is $350 \times 10^{-9} \mathrm{~s}$. If the lifetime is small or comparable to this time then the state can be detected by its decay in flight, and we derive a limit for production independent of the interaction cross section.

$$
\begin{gathered}
\eta \sigma_{\mathrm{P}} \exp (-L / c \tau \gamma \beta)\left\{1-\exp \left[-\left(L_{2}-L\right) / c \tau \gamma \beta\right]\right\} \\
<2.9 / 7.82 \times 10^{44} \mathrm{~cm}^{2}(90 \%),
\end{gathered}
$$

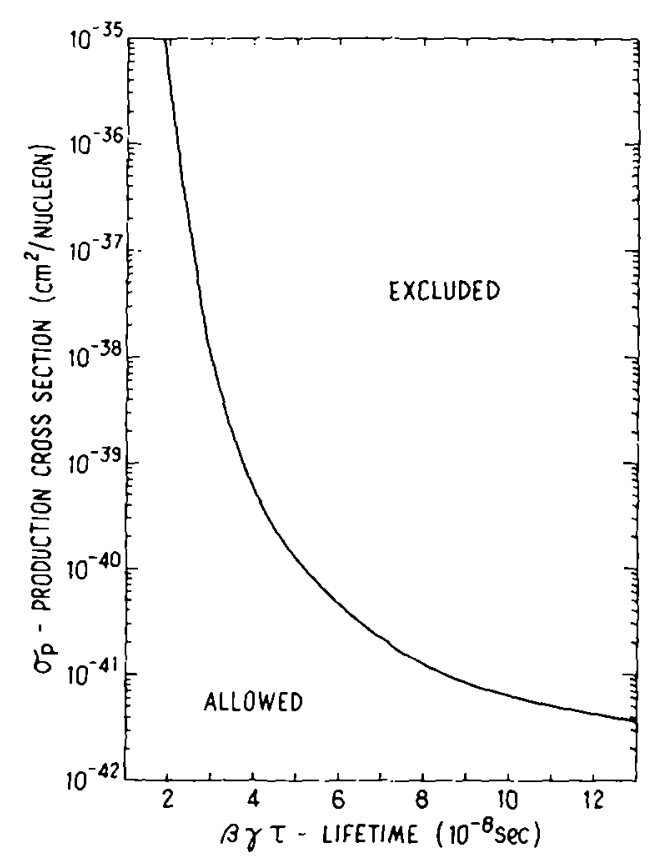

Fig. 1. Limits for axion production and decay in flight. There is little sensitivity below $10^{-8} \mathrm{~s}$.
$L=105 \mathrm{~m}, L_{2}=111.4 \mathrm{~m}, \sigma_{\mathrm{p}}=$ production cross section per nucleon assuming no shadowing. The second term in curly brackets reflects the finite length of the detector. If the axion is very stable, $c \tau \gamma \beta$ large, then it will not decay in the detector.

In fig. 1 we plot the limit curve for $\eta=0.1$. Since for axion masses in excess of $\sim 1 \mathrm{MeV}$ a lifetime of $10^{-10} \mathrm{~s}$ is anticipated we have negligible sensitivity to such states. We can exclude the existence of neutral states with fairly long lifetimes or the production of short lifetime states at large $\gamma$.

Conclusions. We have interpreted our beam dump experiment in terms of the production of new, non neutrino like states. These states would characterize themselves by either a velocity, $\beta<1$ or apparent neutral-current but no charged-current interactions or both.

We observe no slow moving $(\beta<0.89)$ neutral states and find a limit of $<2.2 \%$ heavy states per neutrino produced in the dump into our $10 \mathrm{mrad}$ acceptance. Our limit on new neutral, stable states is, with $\eta=0.1$

$\sigma_{\mathrm{I}} \sigma_{\mathrm{p}}<2.26 \times 10^{-70} \mathrm{~cm}^{4} /$ nucleon $^{2}$.

Our limit on new unstable states is not very restrictive. For $\beta \gamma \tau=5 \times 10^{-8} \mathrm{~s}$ a production cross-section limit of

$o_{\mathrm{p}}<1.18 \times 10^{-40} \mathrm{~cm}^{2} /$ nucleon,

is set. Since the sensitivity here is independent of detector mass but strongly dependent on time of flight the $7 \mathrm{ft}$ bubble chamber (Jacques et al. [5]) may be able to obtain a considerably better limit because of the $43 \mathrm{~m}$ flight distance to the bubble chamber and its larger geometric acceptance.

To compare our result on stable light states to other limits we will use the value $\eta=0.01$ in eq. (7) as previous searches have assumed. This gives:

$\sigma_{\mathrm{I}} \sigma_{\mathrm{p}}<2.26 \times 10^{-69} \mathrm{~cm}^{4} /$ nucleon $^{2}$

This is better than the previous limits $[5,6]$ by at least a factor of 5.5 .

We would like to thank M. Bregman, M. Claudson, L. Rivkin, J. Roeder, S. Russek, P. Timbie, M. Yudis, T.A. Gabriel and M. Levine for assistance. This work was supported by the US Department of Energy under contracts EY-76-C-02-0016 and DE-AC02-76ERO3064. 


\section{References}

[1] H. Faissner, Proc. 1980 Neutrino Conf., to be published; Particle Data Group, Rev. Mod. Phys. 52 (1980) \$1 p. S115.

[2] R. Peccei and H. Quinn, Phys. Rev. Lett. 38 (1977) 1440; S. Wcinberg, Phys. Rev. Lett. 40 (1978) 223;

F. Wilczek, Phys. Rev. Lett. 40 (1978) 279;

S. Dimopoulos, S. Raby and Kane, Univ. of Michigan preprint UM HE 80-22, to be published.
[3] A. Soukas et al., Phys. Rev. Lett. 44 (1980) 564.

[4] A. Entenberg et al., Phys. Rev. Lett. 42 (1979) 1198, and references therein.

[5] P. Coteus et al., Phys. Rev. Lett. 42 (1979) 1438; P.F. Jacques et al., Phys. Rev. D21 (1980) 1206; for a comparison see: J. LoSecco, Proc. XXth Intern. Conf on High energy physics (1980), AIP Conf. Proc. series, to be published.

[6] H. Faissner et al., Phys. Lett. 60B (1976) 401.

[7] M. Bourquin and J.M. Gaillard, Nucl. Phys. B114 (1976) 334. 